\title{
Cross-modal transfer of oddity learning
}

DONALD J. TYRRELL FRANKLIN AND MARSHALL COLLEGE

College students were given visual or tactual training on either a double stimulus oddity problem or a two-choice texture discrimination. Cross-modal transfer (CMT) was measured by performance on a subsequent oddity problem in the alternate sense mode. CMT was evident following oddity but not discrimination training.

Cross-modal transfer (CMT) of training, the integration of information received through different sensory modes, has been demonstrated in children (Houck, Gardner, \& Ruhl, 1965) and adults (Gaydos, 1956). Blank \& Bridger (1964) have drawn a distinction between cross-modal equivalence (CME), which involves recognition of a particular stimulus presented in different sense modalities, and crossmodal concept ( $\mathrm{CMC}$ ), which requires utilization of a concept in solving problems involving dissimilar yet analogous stimuli presented in distinct sensory modes. Blank \& Bridger (1964) were able to demonstrate $\mathrm{CME}$ in young children when comparing visual and haptic sensory inputs and CMC employing visual and auditory sense modes. They were not able to demonstrate CMC between visual and tactual problems presented to deaf and hearing children (Blank \& Bridger, 1966). The present experiment was designed in an attempt to demonstrate $\mathrm{CMC}$ between visual and tactual problems presented to college students. Design

Ss, 40 college freshmen, were given either visual or tactual training. One half the Ss in each modality were presented with a double stimulus oddity problem and the remaining half were given a two choice texture relevant discrimination problem. Following the training problem, all visual Ss were presented with a tactual oddity problem, and all tactual Ss were given a visual oddity problem. Cross-modal transfer of the oddity concept would be indicated if the final oddity problem were solved more rapidly following oddity training than following either type of discrimination training, or more rapidly than oddity learning within the same modality during the training phase. Apparatus

A portable version of the WGTA was modified to accommodate interchangeable stimulus trays appropriate for visual and tactual stimulus presentation. Visual problems were presented with stimuli placed over the reward wells of a conventional sliding tray. $\mathrm{S}$ responded by pushing aside the stimulus of his choice until the reward well was uncovered. Ss were instructed to respond by pushing the base upon which the stimulus was mounted, thereby eliminating any relevant tactual learning during the visual problem.
For tactual problems the tray was replaced by a box, the floor of which was identical to the sliding visual tray. The side of the box facing $S$ had two cloth draped response holes, 6 in. in diameter, drilled directly in line with and 2 in, above the reward wells. The side of the box facing $E$ was open, providing easy access to the reward wells. Tactual trials consisted of $\mathrm{S}$ placing his hands through the response holes, tactually investigating the stimulus objects placed over the wells, pushing one of these objects aside, and feeling the uncovered well for the reward.

The stimuli presented on any one oddity trial were two pairs of solid geometric forms. Each pair was mounted on a gray $4 \times 4$ in. Masonite base. The stimulus pairs were constructed from a pool of six basic geometric forms which were equal in volume $\left(1 \mathrm{in} .{ }^{3}\right)$. The six forms were divided into separate sets of three forms each (Set $1=$ cube, sphere, and pyramid; Set $2=$ cylinder, rectangle, and cone). The pairs were formed by combining each form of a set with each of the other forms of the set. This produced three pairs having two identical forms (e.g., two cubes) and three pairs having odd forms (e.g, cube and sphere) within each set. Nine different combinations of one similar and one odd pair were formed within each basic set, and one of these combinations was presented on each oddity trial.

The stimuli for the visual and tactual discrimination problems were two cylinders, each mounted on a separate gray Masonite base. One of these had circles of sandpaper $1 / 4 \mathrm{in}$, in diameter glued over all exposed surfaces. The other was smooth except for two strips of sandpaper $1 / 8 \mathrm{in}$. thick around the sides. This discrimination was a texture discrimination with form constant within and between trials.

\section{Procedure}

Each S, upon appearance, was randomly assigned to the visual or tactual training condition and presented either an oddity or a discrimination task within that modality. For each oddity $S$ one of the stimulus sets was chosen, and one of the nine two pair combinations was presented on each trial. All combinations were presented randomly with the restriction that no combination was repeated on consecutive trials and no pair of a combination could be present on more than two consecutive trials. Following each choice of the odd pair a poker chip was found in the reward well. Ss were run to a criterion of 10 consecutive correct responses; the noncorrection technique was employed and position was randomized according to the Gellermann series. The texture discrimination was handled in a similar fashion 
with the exception that the same two stimuli were employed on every trial and one of the textures was randomly made positive for each $S$.

Following criterion on the training problem the apparatus was appropriately altered and the $S$ was immediately presented with an oddity problem. The stimulus set employed for the second oddity problem was the one not used during original oddity training for the oddity trained Ss, and was determined randomly for those Ss trained on the discrimination problem. All features were identical to the training problem. Trials were continued until a criterion of 10 consecutive correct responses was attained. Results

A Mann-Whitney $U$ test on errors to criterion during training indicated no differences between the visual and tactual oddity performance $(U=47, p>.10)$ or between visual and tactual discrimination learning $(U=31, p>.10)$. Similar comparisons of errors to criterion on the transfer oddity problems indicated that visual oddity performance following tactual oddity training was significantly better than visual oddity following tactual discrimination training $(U=25, p<.05)$ or visual oddity during training $(\mathrm{U}=10, \mathrm{p}<.002)$. Similarly, tactual oddity following visual oddity training was more efficient than tactual oddity following visual discrimination training $(U=20, p<.025)$ or tactual oddity during training $(\mathrm{U}=19, \mathrm{p}<.01)$.

A comparison of errors on visual oddity performance following tactual oddity training with tactual oddity following visual oddity training indicated that the visual problem during transfer was easier than the comparable tactual problem $(U=25, p<.05)$.

\section{Discussion}

The decrease in errors in solving an oddity problem when given relevant oddity training compared to that following either no training or irrelevant discrimination training is positive evidence that college students can employ a concept in one modality after forming that concept in a different modality. This CMC was evident from visual to tactual problems and vice versa. The finding of fewer errors on the visual transfer than on the tactual transfer indicates an apparent difference in the degree of transfer. More information about a visual problem is acquired tactually than is acquired about a tactual problem during visual training. That this performance increase is true CMC and is not amenable to a more general learning set explanation is strengthened by the lack of facilitation of oddity performance following the discrimination training.

\section{References}

BLANK, M., \& BRIDGER, W. H. Cross-modal transfer in nursery-school children. J. comp. physiol Psychol, 1964, 58, 277-282.

BLANK, M., \& BRIDGER, W. H. Conceptual cross-modal transfer in deaf and hearing children. Child Develpm., 1966, 37, 29-38.

GAYDOS, H. Intersensory transfer in the discrimination of form. Amer. J. Psychol, 1956, 69, 107-110.

HOUCK, E. V., GARDNER, G., \& RUHL, D. Effects of auditory and visual pretraining on performance in a tactile discrimination task. Percept. mot. Skills, 1965, 20, 1057-1063.

\section{Note}

1. This investigation was supported in part by the Oklahoma State University Research Foundation. The author wishes to thank Miss Peggy Gouge and Miss De Miner for their assistance in running Ss. 